\title{
Reference values for metacarpophalangeal joint stiffness in normals
}

\author{
A HOWE. ${ }^{1}$ D THOMPSON, ${ }^{2}$ V WRIGHT
}

From the ${ }^{1}$ Rheumatism Research Unit, University Department of Medicine, General Infirmary at Leeds; and the ${ }^{2}$ Faculty of Technology (Eng. Mechs.), The Open University, Milton Keynes

SUMMARY A new form of microprocessor-controlled arthrograph is described which measures stiffness parameters of the metacarpophalangeal joints of the index, middle, and ring fingers of both hands. The apparatus is simple and quick to use and gives reproducible results. The arthrograph is used to determine the reference limits for stiffness at the middle finger of the right hand in 128 normal subjects. It is found that incorporating information on the subject's finger circumference allows a much improved precision to be achieved, but the precision is not further enhanced by including details of age and sex.

Key words: arthrograph, microprocessor.

The importance of joint stiffness as an aid to the diagnosis and management of rheumatoid arthritis and other connective tissue disorders is well known. The need for some method of objectively assessing the stiffness has also been extensively stated in the literature, and a number of mechanical devices such as arthrographs for the measurement of joint stiffness have been described over the past 25 years. ${ }^{1-5}$ Devices have been built to measure stiffness at both the metacarpophalangeal (MCP) and knee joints, and useful results have been obtained in research environments. However, despite this research activity, there has been no major therapeutic trial in which objective stiffness measurements have been used. This is due, at least in part, to the difficulty of obtaining data quickly and repeatedly in a clinical environment. The arthrographs reported have been primarily research and not clinical tools, requiring the skills of a practised experimenter to obtain useful results, which require tedious analysis to reveal the parameters used to compare and monitor stiffness.

It was apparent that the worth of objective stiffness measurements could not be fully evaluated until there was an arthrograph which would not only be adaptable for use in a clinic but would produce results of sufficient interest to persuade clinicians to

Accepted for publication 9 January 1985.

Correspondence to Dr A Howe, Rheumatism Research Unit, 36 Clarendon Road, Leeds LS2 9PJ. use it of their own volition. The purpose of the present study was to develop a new arthrograph which would be suitable for both research and clinical environments and then to use it to study the stiffness of both normal and diseased joints so as to relate these findings to the symptoms of which the sufferers complain. This paper describes the arthrograph and its use in the determination of a range of reference values from 128 normal subjects.

\section{REFERENCE VALUES}

Reference or normal ranges are useful in the first phase of the diagnostic process. When the measured value does not fit the pattern of values found in the reference population (e.g. healthy persons) it usually indicates closer examination of the patient. The choice of reference populations should reflect the characteristics of the target population as closely as possible. The most appropriate values would come from people who have been declared healthy after careful examination, but in this study as in others the data have been obtained from the most readily available source and included a higher proportion of medical and academic personnel than would generally be found in an epidemiological study of rheumatoid patients. The precision of the reference values is also determined by the size of the reference population. Bezemer et al. ${ }^{6}$ recommended a sample size of over 50 and preferably more than 100 people in each group. They also suggest using as 
reference values the limits which remain after removing the top and bottom 2.5 and $10 \%$ percentiles from the collected data, and this is the method that has been adopted in this study. The choice of more than one limit on either side emphasises the empirical nature of reference limits, which should not be treated as immutable values that declare a patient to be 'normal' or 'abnormal' in isolation from other symptoms and medical opinion.

\section{ARTHROGRAPH DESIGN CRITERIA}

To be of maximum usefulness an arthrograph should be able to operate equally well in both research and clinical environments. For the development of a rheological model for the joint it is necessary for the machine to be capable of applying one of a number of different waveforms of variable amplitude and frequency. For clinical acceptance the arthrograph must be sufficiently small and portable to allow its use in the often confined spaces found on the hospital ward and clinic, and the results it produces should be available immediately in a form which is meaningful and useful.

Recent developments in microprocessor technology allow the construction of an arthrograph which will satisfy these criteria without being restrictively complex or expensive. For reasons of size and ease of patient relaxation it was decided to concentrate on a machine for the measurement of stiffness at the MCP joint.

\section{FINGER ARTHROGRAPH}

The arthrograph comprises three main components: the drive system, limb support and torque transducer, and the microprocessor.

\section{Drive system}

The drive assembly is based around a PML, G9M4 DC servomotor with integral tachometer feedback and amplifier. When these are used with position feedback it is possible to move the motor armature in phase with an electrical waveform fed into the amplifier. This does not require a gearbox or other mechanical coupling and so eliminates mechanical backlash in the system and also allows the very rapid accelerations found in square and step waveforms to be reproduced more accurately.

\section{Limb support and torque transducer}

The finger holder is a split plastic tube bolted onto a strain gauged lever arm which is mounted directly onto the shaft of the motor. The finger to be tested is placed in the finger holder, with the forearm prone on the upper surface of the arthrograph. When positioned correctly the finger may be secured very firmly by tightening the tube onto the

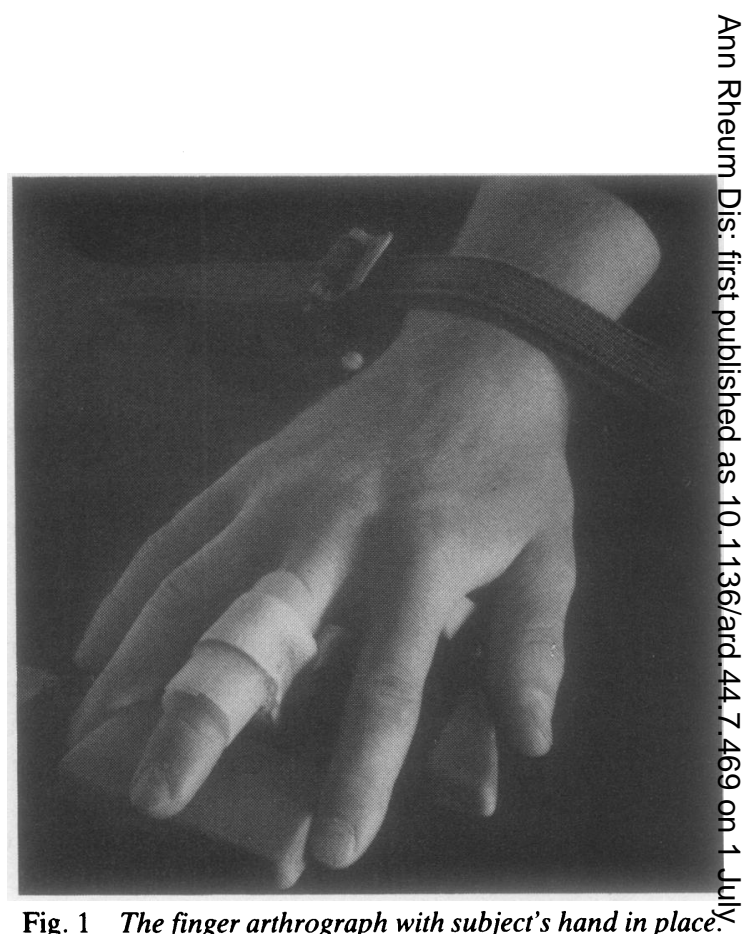

Fig. 1 The finger arthrograph with subject's hand in place.

proximal phalangeal joint by means of a Velcro strap. Finger and thumb rests are provided for thoses digits not under test (Fig. 1). The machine was buils to measure stiffness in an horizontal plane, in ordef르 to eliminate torque arising from the weight of the finger. Moving the finger in adduction/abduction makes it possible to measure stiffness in the MCP joints of the index, middle, and ring fingers of both hands.

\section{Microprocessor}

The microprocessor hardware is a Z80 systen consisting of a Comart $\mathrm{C} 100$ Communicator with twin floppy disk drives, an IO Technology 12-bi DAC board, a visual display unit, and dot-matrix printer. The control program is written in PASCAI and $C$ and provides an automatic test mode, in which the selection of test conditions and supervi sion of the test is left entirely to the microprocessors and a manual mode, which allows the operator to select the test waveform (sine, triangular, square, of step), frequency $(0.01-3.0 \mathrm{~Hz})$, amplitude, and offset. The computer disallows combinations amplitude and frequency which are likely to cause undue distress to the patient, or introduce inertia effects into the results.

Principle of operation

For clinical use the sinusoidal waveform is the mos $\mathbb{Q}$ appropriate, as this is least likely to promote spasti city. The triangular waveform is of use for investigas tion of the characteristics of the joint when subject 
to a constant strain rate. It produces hysteresis loops which are very similiar to those obtained with a sinusoidal waveform, though reduced in area. The square and step waveforms are important in the investigation of stress-relaxation properties in the joint and can be used to estimate the relaxation time constant. When the test is started the arthrograph first finds the equilibrium position of the joint, defined here as the midpoint between those two positions at which the torques necessary to maintain a constant rate of strain are equal. Tests are carried out relative to this datum, allowing direct comparison of results from different subjects and reducing the errors arising from inaccurate positioning of the hand. The chosen test waveform is calculated in digital form and fed to the servoamplifier via a digital-to-analogue converter. The instantaneous position and resistance to torque are collected from the transducers and monitored by the computer. Each successive loop is compared with the previous one until the results are repeatable, at which point the test is stopped. For normal subjects who do not exhibit the pronounced articular gelling of rheumatoid and osteoarthrosis patients the first two cycles are often repeatable. If the subject has difficulty in relaxing, then it may take three or four cycles to become familiar with the sensation before repeatable loops are obtained. The stiffness characteristics are essentially unchanged by cycling at low frequencies and amplitudes for the few seconds necessary for the test. The operator is offered the choice of repeating the test, choosing a new test, or analysing the stored data. The analysis involves calculating the area and mean slope of the loop and presenting these data together with a facsimile of the loop on paper for inclusion in patients' records.

A typical hysteresis loop is shown in Fig. 2. As indicated, the loop may be divided into three distinct regions. The two peripheral areas give information on the range of motion and laxity of the joint, but for stiffness measurements the central region is of more interest. When a standard test amplitude of $4^{\circ}$ is adopted at the equilibrium position hysteresis loops similar to that shown in Fig. 3 are obtained. The loop may be characterised by two parameters: the mean slope of the loop and its area (corresponding to the energy dissipated each cycle). As the area is closely linked to the slope of the loop,

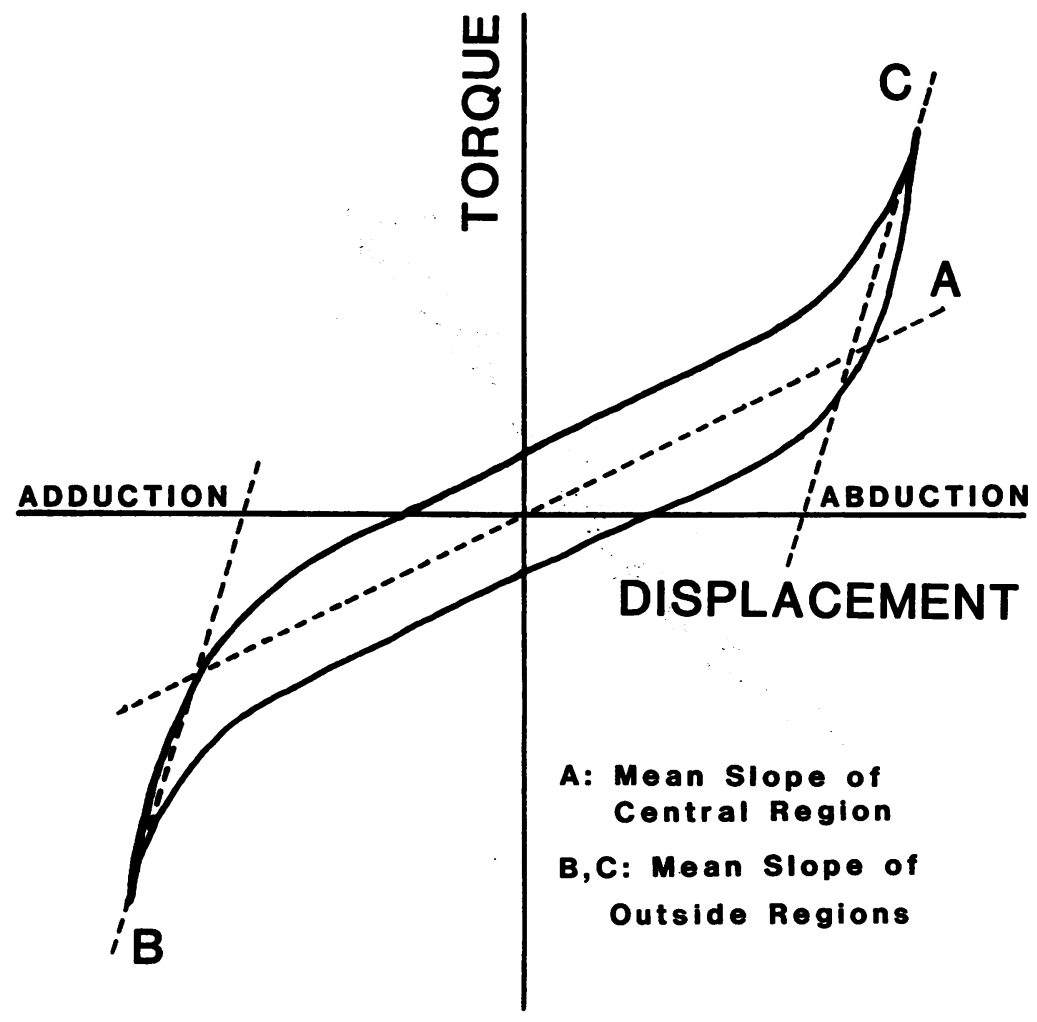

Fig. 2 A typical hysteresis loop obtained from a normal joint. 


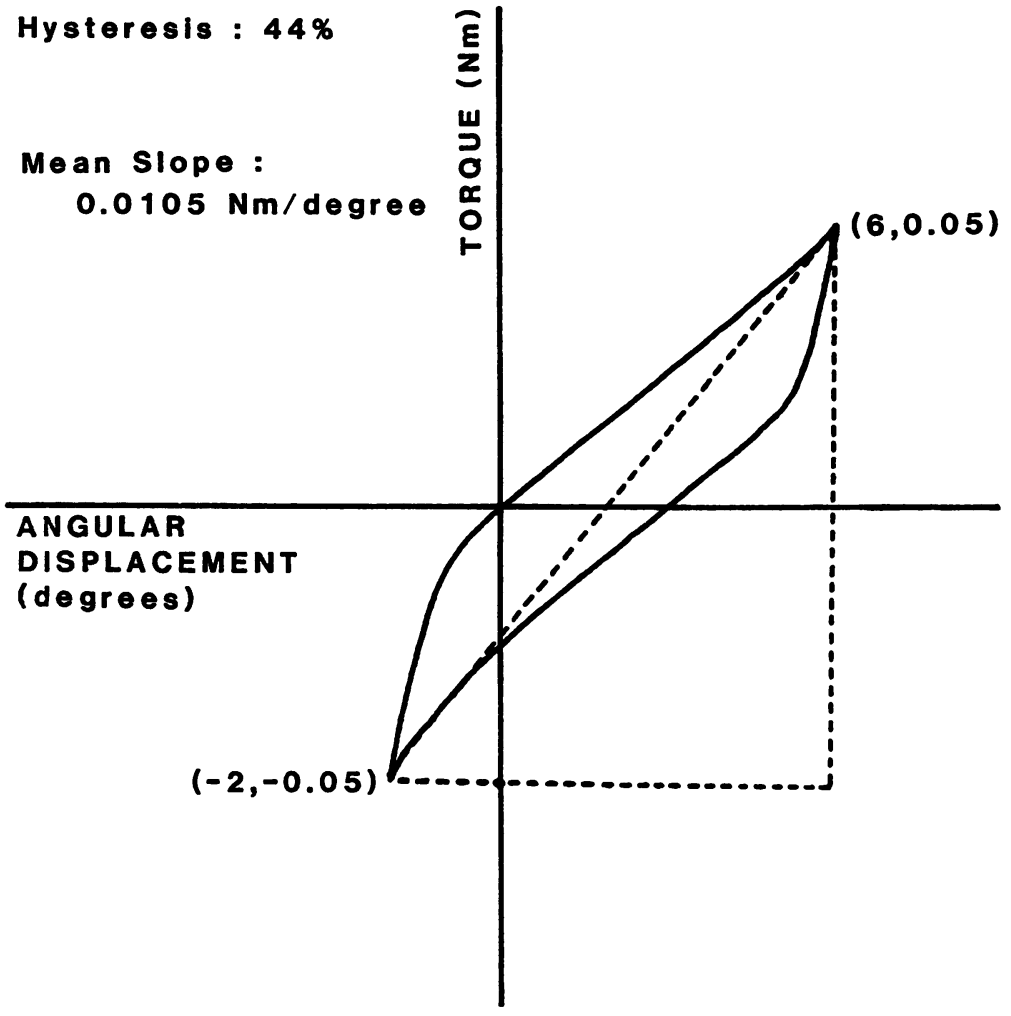

Fig. 3 Hysteresis loop obtained from a 22 year old normal male $\overline{2}$ using the standard test procedure + (4 amplitude, $0.2 \mathrm{~Hz}$ frequency at the equilibrium position).

it is normalised by expressing it as a percentage of the triangle fitted to the loop as shown in Fig. 3. Repeatability tests were undertaken and showed that coefficients of variation below $5 \%$ could be expected in the measurement and calculation of these two parameters. With the automatic mode of testing the entire test can be carried out at the press of a single button. The need for skilled operators and lengthy analysis of hysteresis loops is eliminated, encouraging the frequent use of the device in the clinic.

\section{Materials and methods}

Tests were conducted on the middle finger of the right hands of 144 subjects, equally divided between the sexes. The subjects ranged in age from 10 to 83 years and were either conscripted from the staff and students of Leeds University or were visitors attending the University Open Day. Before the test began each subject was made comfortable, and the hand was placed on the arthrograph so that the centre of rotation of the MCP joint coincided with that of the motor shaft. As there is no power to the motor at this stage of the test, the finger may be moved freely in the machine to aid alignment. The hand was supported under adjacent MCP joints, and the armo rested on a bean bag, with the wrist straight. The arm and metacarpal bones of the test finger were aligned parallel with the long axis of the arthrograph and when in place were stabilised with adjustable? straps. The subjects were asked to relax as much as possible, at which point the test was carried out. The 3 test waveform was a sine wave of amplitude $4^{\circ}$ and 9 frequency $0.2 \mathrm{~Hz}$, and the tests were conducted by the automatic mode. It had been found that nearly을. everybody was able to relax during tests at this N frequency. The test lasted about 90 seconds, after ${ }^{\text {T }}$ which a further three minutes were required for $N$ analysis of the data. Details of age, sex, and $\mathbb{W}^{N}$ dominant hand were noted for each subject. In addition an estimation of the tissue bulk around the joint was made by measuring the circumference of $\frac{\text { }}{\overparen{D}}$ the finger close to the webs.

\section{Results}

The size of each of the various anthropometric $\frac{\mathbb{D}}{\mathbb{D}}$ groups used in this study is shown in Table 1. Details of dominant hand and finger circumference were 
Table 1 Numbers of subjects in each of the anthropometric groups

\begin{tabular}{lrcc}
\hline & Male & Female & Total \\
\hline Left-handed & 4 & 4 & 8 \\
Right-handed & 54 & 42 & 96 \\
Other/unknown & 6 & 18 & 24 \\
Total & 64 & 64 & 128 \\
\hline
\end{tabular}

only collected from 104 of the subjects, and 16 subjects were unable to relax sufficiently to produce satisfactory results. Although a trained operator would be able to extract useful information under such circumstances, in this study no data were collected from these subjects.

Table 2 shows the reference values obtained from the measured data; separate values are shown for male and female subjects where these differed significantly. Table 3 summarises the significance of the correlations obtained for combinations of arthrograph and anthropometric data. Significant correlations were obtained for mean slope with age, mean slope and hysteresis with finger circumference, and finger circumference with age.

Table 2 Reference values for stiffness measurements

\begin{tabular}{lll}
\hline & \multicolumn{2}{l}{ Percentiles } \\
\cline { 2 - 3 } & 2.5 and $97.5 \%$ & 10.0 and $90.0 \%$ \\
\hline Hysteresis (\%) & $22-58$ & $26-49$ \\
Mean slope (Nm/deg) & (M) $0.0028-0.0226$ & $0.0056-0.0160$ \\
& (F) $0.0023-0.0143$ & $0.0029-0.0095$ \\
Equilibrium position (deg) & $(-9)-4$ & $(-7)-1$ \\
Finger circumference (mm) (M) 55-71 & $57-69$ \\
& (F) 53-62 & $54-60$
\end{tabular}

Separate values are only shown for male and female subjects where these differ significantly.
Table 4 Results of the multiple regression analyses

Dependent variable: mean slope

Regression due to age: $F=8 \cdot 8055^{*}$

Additional regression due to finger circumference: $F=35.9742^{*}$

Regression due to finger circumference: $F=47.9631^{*}$

Additional regression due to age: $F=0.6133$ (NS)

Dependent variable: hysteresis

Regression due to age: $F=0 \cdot 1498$ (NS)

Additional regression due to finger circumference: $F=7.4079^{*}$

Regression due to finger circumference: $\mathrm{F}=7 \cdot 0622^{*}$

Additional regression due to age: $F=0.5441$ (NS)

Dependent variable: equilibrium position

Regression due to age: $F=1 \cdot 8780$ (NS)

Additional regression due to finger circumference: $F=0.0730$ (NS)

Regression due to finger circumference: $F=0.6377$ (NS)

Additional regression due to age: $\mathrm{F}=1.2909$ (NS)

${ }^{*} \mathrm{p}<0 \cdot 01 ; \mathrm{NS}=$ not significant.

Covariance analysis with age as a covariable showed a significant difference in the mean slopes of male and female subjects.

Multiple linear regression on age and finger circumference showed that finger circumference is the single dominating influence on both mean slope and hysteresis (Table 4), and Figs 4 and 5 show the regression and parallel percentile lines for the variation of mean slope and hysteresis with finger circumference.

\section{Discussion}

A clear relationship between age and mean slope at the MCP joint has emerged from this work. A similar relationship for the hysteresis has not been observed. Such et al. ${ }^{7}$ investigated knee stiffness in over 60 subjects between the ages of 21 and 65 and found no correlation between elastic stiffness and age, though the energy dissipation did show a

Table 3 Summary of the significance of the correlations obtained for combinations of stiffness and anthropometric data

\begin{tabular}{|c|c|c|c|c|c|c|c|c|c|c|}
\hline \multicolumn{2}{|l|}{ Variables } & \multicolumn{3}{|l|}{ All } & \multicolumn{3}{|c|}{ Female } & \multicolumn{3}{|l|}{ Male } \\
\hline Independent & Dependent & Total & $\begin{array}{l}\text { Left } \\
\text { handed }\end{array}$ & $\begin{array}{l}\text { Right } \\
\text { handed }\end{array}$ & Total & $\begin{array}{l}\text { Left } \\
\text { handed }\end{array}$ & $\begin{array}{l}\text { Right } \\
\text { handed }\end{array}$ & Total & $\begin{array}{l}\text { Left } \\
\text { handed }\end{array}$ & $\begin{array}{l}\text { Right } \\
\text { handed }\end{array}$ \\
\hline Age & Slope & $*$ & - & - & - & - & * & $*$ & - & - \\
\hline Age & Hysteresis & - & - & - & - & - & - & - & - & - \\
\hline Age & Equilibrium position & - & - & - & - & $*$ & - & - & - & - \\
\hline Finger circumference & Slope & $t$ & - & $t$ & $*$ & - & - & $\dagger$ & - & $*$ \\
\hline Finger circumference & Hysteresis & $*$ & - & $*$ & - & - & - & - & - & - \\
\hline Finger circumference & Equilibrium position & - & - & - & - & - & - & - & - & - \\
\hline Age & Finger circumference & $\dagger$ & - & $\dagger$ & - & - & * & $\dagger$ & - & $\dagger$ \\
\hline
\end{tabular}

- =not significant; ${ }^{*} p<0.01 ;+p<0.001$. 


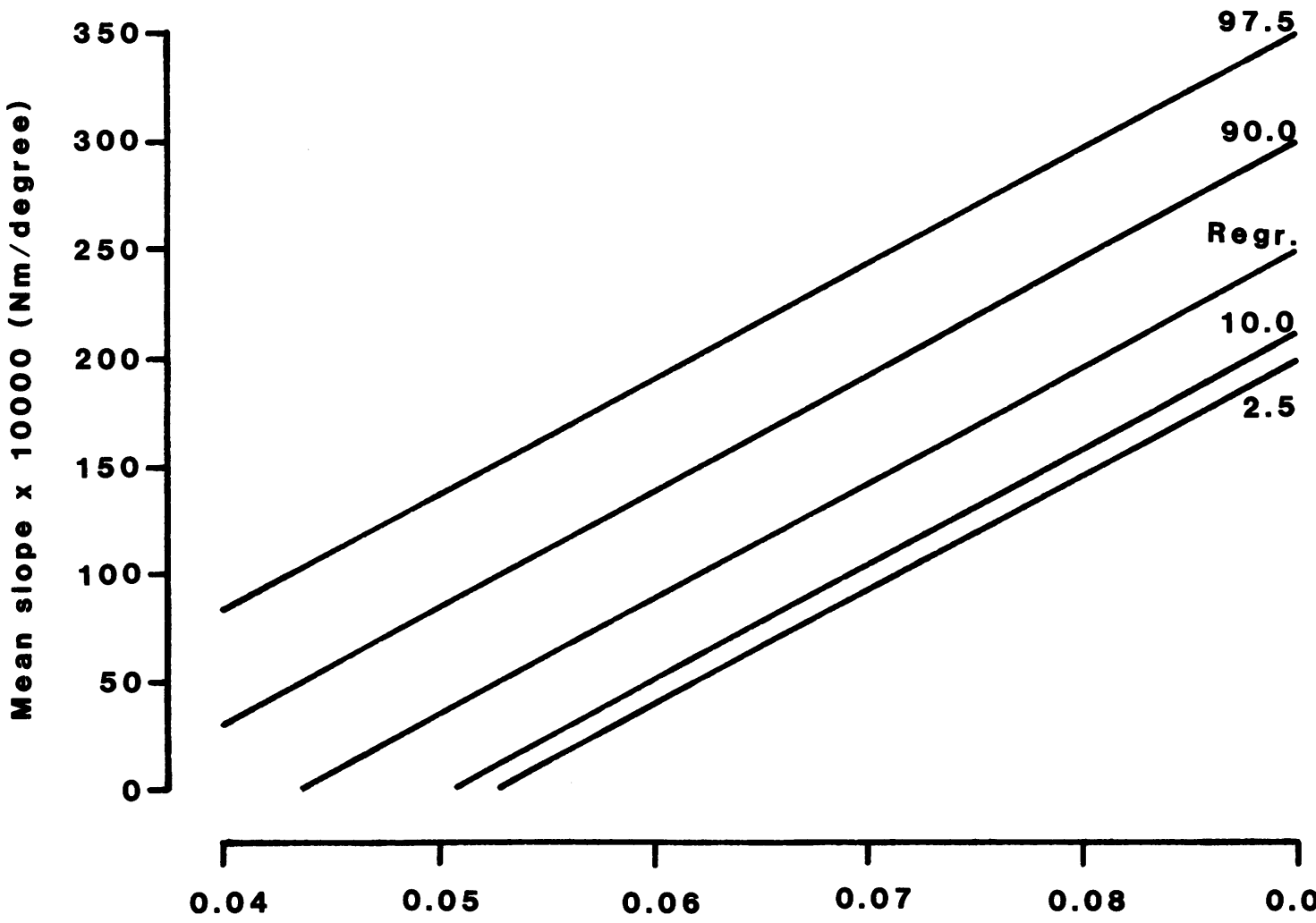

Finger circumference $(m)$

Fig. 4 Linear regression and parallel percentile lines for mean slope with finger circumference.

significant increase with age. Thompson et al. ${ }^{4}$ could find no relationship between either elastic stiffness or energy dissipation and age. Wright and Johns ${ }^{8}$ suggested that elastic stiffness increased with age at the MCP joint, but the small sample size did not allow any significance to be attached to the result.

A relationship between stiffness and the tissue bulk around the joint (measured as finger circumference) has also been shown in this study. This is in agreement with the work of Wright and Johns ${ }^{1}$ on a cat wrist joint, which showed that passive extension of the muscle accounted for $41 \%$ of the total stiffness, with a further $47 \%$ coming from the joint capsule. Such et al. ${ }^{7}$ and Thompson et al. ${ }^{4}$ measured thigh girth and obtained a positive correlation between this and both elastic stiffness and dissipated energy. At the MCP joint Unsworth et al. ${ }^{9}$ found a correlation between subject mass and the elastic stiffness and energy dissipation. A negative correlation was obtained in this study for the relationship between finger circumference and hysteresis. This is evidence for a more rapid increase of mean slope than energy dissipation with increasing finger circumference, perhaps implying that the hysteresis includes a significant component other than the tissue bulk.

The difference between the stiffness of male and $\rightarrow$ female subjects has been noted by many workers, with results showing that on average females exhi- N bited between 55 and $70 \%$ of the male stiffness at both the MCP and knee joints. ${ }^{1457}$ Such et al. ${ }^{7} \mathrm{~N}$ thought that this difference was greater than could $\omega$ be accounted for by size alone, but no other workers? found evidence for this.

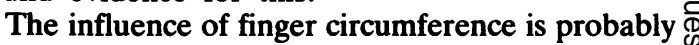
sufficient to explain the differences observed be-? tween measurements of dominant and non- $T$ dominant hands. The quantity of data collected for $\frac{}{\mathrm{D}}$ non-dominant hands is, however, too small for any $\stackrel{P}{P}$ significance to be attached to the results from this $\bigcirc$ study.

The relationship between age and finger cir- 


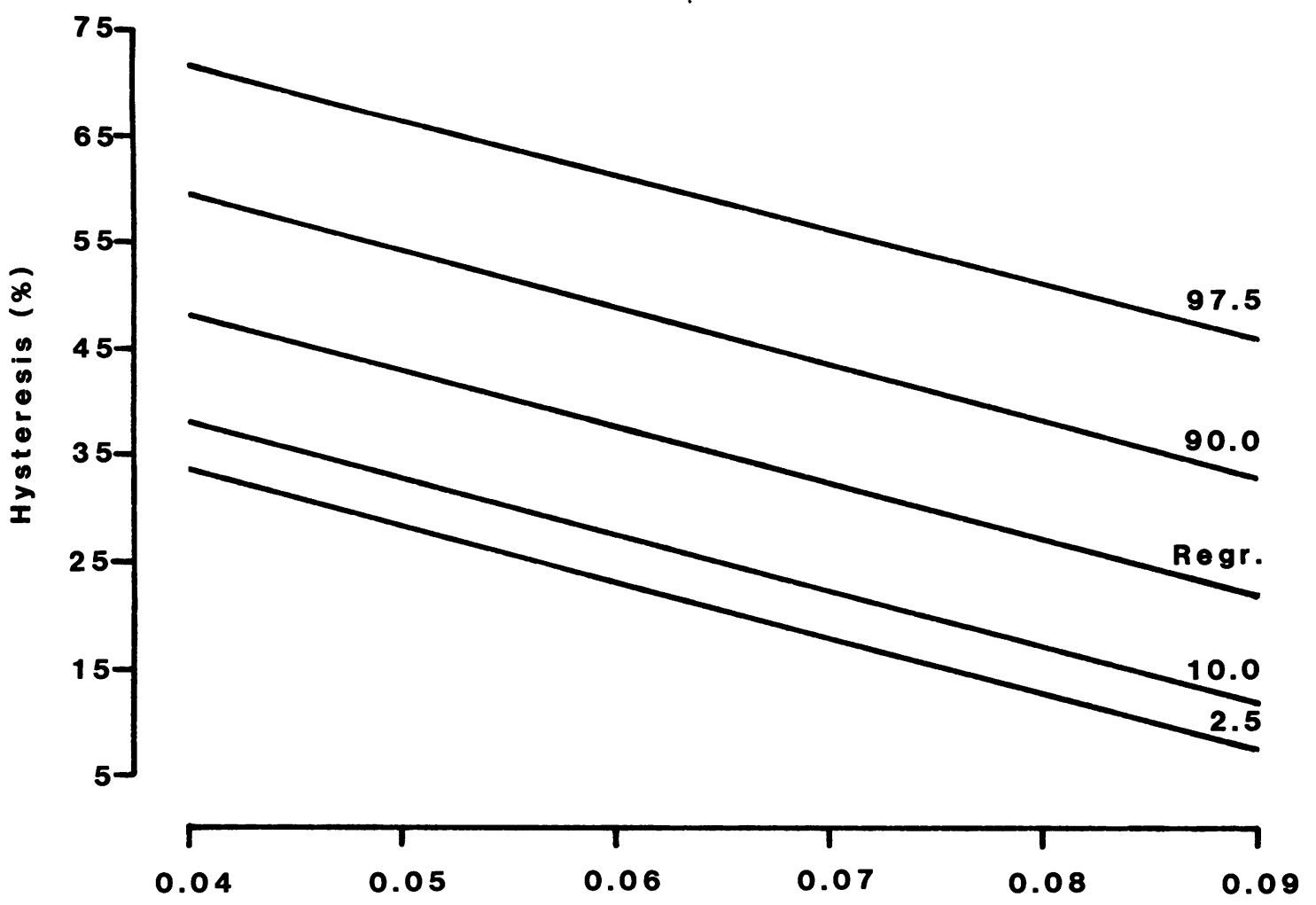

Finger circumference $(m)$

Fig. 5 Linear regression and parallel percentile lines for hysteresis with finger circumference.

cumference has not been reported before in this context either at the knee or MCP joint and is perhaps a little surprising. Although a correlation might be expected during the active growing years of puberty, it is not clear as to the cause of the increase in finger circumference after this time. It is suggested that the increase is a result of usehypertrophy, and there is circumstantial evidence for this in the reduced correlations obtained for lefthanded and female subjects. It is known that the increase in size of dominant over the non-dominant hand is of both genetic and environmental origin, and the phenomenon noted here could be a manifestation of this. The result is of importance as it is found in the multiple regression analyses that the correlation observed for the increase of mean slope with age and the differences observed between the sexes can be satisfactorily accounted for by the differences in finger circumference alone.

Since normal limits have been established, a datum now exists with which to compare results obtained with the arthrograph on diseased patients. It is hoped that this will include the study of patients with rheumatic and connective tissue disorders, evaluation of the relief of stiffness by intra-articular therapy, surgery and systemic drug therapy, and correlation of pharmacokinetic drug profiles with a quantitative assessment of stiffness.

The authors wish to acknowledge the support of the Arthritis and Rheumatism Council who enabled this work to be undertaken.

\section{References}

1 Wright V, Johns R J. Observations on the measurement of joint stiffness. Arthritis Rheum 1960; 3: 328-40.

2 Long C, Thomas T, Crochetiere W J. Objective measurement of muscle tone in the hand. Clin Pharmacol Ther 1964; 5: 909-17.

3 Backlund L, Tiselius P. Objective measurement of joint stiffness in rheumatoid arthritis. Acta Rheumatol Scand 1967; 13: $275-88$. 


\section{Howe, Thompson, Wright}

4 Thompson D T, Wright V, Dowson D. A new form of knee arthrograph for the study of stiffness. Eng Med 1978; 7: 84-92.

5 Unsworth A, Yung P, Haslock I. Measurement of stiffness in the metacarpophalangeal joint: the arthrograph. Clin Phys Physiol Meas 1982; 3: 273-81.

6 Bezemer P D, Netelnbos J C, Mulder C, Theune J A, Stamhuis I $\mathbf{H}$, Straub J P. Determining reference (normal) limits in medicine: an application. Stat Med 1983; 2: 191-8.
7 Such C H, Unsworth A, Wright V, Dowson D. Quantitative study of stiffness in the knee joint. Ann Rheum Dis 1975; 34: 286-91.

8 Wright $\mathrm{V}$, Johns R J. Physical factors concerned with the stiffness of normal and abnormal joints. John Hopkins Med J1960; 106: 215-31.

9 Unsworth A, Bey P M A, Haslock I. Stiffness in the metacarpophalangeal joints of young adults. Clin Phys Physiol $\frac{\mathrm{N}}{2}$ Meas 1981; 2: 191-8. 